J. Appl. Math. \& Informatics Vol. 31(2013), No. 5 - 6, pp. 711 - 732

http://dx.doi.org/10.14317/jami.2013.711

\title{
DEFAULTABLE BOND PRICING USING REGIME SWITCHING INTENSITY MODEL
}

\author{
STÉPHANE GOUTTE ${ }^{* \dagger}$ AND ARMAND NGOUPEYOU ${ }^{ \pm}$
}

\begin{abstract}
In this paper, we are interested in finding explicit numerical formulas to evaluate defaultable bonds prices of firms. For this purpose, we use a default intensity whose values depend on the credit rating of these firms. Each credit rating corresponds to a state of the default intensity. Then, this regime switches as soon as one of the credit rating of a firm also changes. Moreover, this regime switching default intensity model allows us to capture well some market features or economics behaviors. Thus, we obtain two explicit different formulas to evaluate the conditional Laplace transform of a regime switching Cox Ingersoll Ross model. One using the property of semi-affine of the model and the other one using analytic approximation. We conclude by giving some numerical illustrations of these formulas and real data estimation results.
\end{abstract}

AMS Mathematics Subject Classification : 60H10, 91G40, 91G60, 91B28, $65 \mathrm{C} 40$.

Key words and phrases : Defaultable bond, Regime switching, Conditional Laplace Transform, Credit rating, Markov copula.

\section{Introduction}

In an economic crisis situation where the credit ratings of countries or firms have a big impact in the general financial market, we need to understand and capture the change of these ratings in the dynamic of firms bond price. Moreover, we also have to model the contagion risk due to a bad rating of a firm with respect to another one. For example, the Bond of countries in the Euro zone are affected by the bad credit rating of Greece. In the literature, models for pricing defaultable securities have been introduced by Merton [23]. It consists of explicitly linking the risk of default and the value of the firms. Although this model is a good issue to understand the default risk, it is less useful in

Received June 22, 2012. Revised November, 27. 2012. Accepted December 5, 2012. ${ }^{*}$ Corresponding author. ${ }^{\dagger}$ Supported by the FUI project $R=M C^{2}$. ${ }^{ \pm}$Supported by ALMA Research.

(C) 2013 Korean SIGCAM and KSCAM. 
practical applications since it is too difficult to capture all the macroeconomics factors which appear in the dynamics of the value of the firms. Hence, Duffie and Singleton [9] introduced the reduced form modeling, followed by Madan and Unal [22], Jeanblanc and Rutkowski [20] and others. The main tool of this approach is the "default intensity process" which describes in short terms the instantaneous probability of default. Moreover to deal with contagion risk, the most popular approach is copula. The credit rating of each firm is modeled by a Markov chain on which we will construct our copula. In this regard, we use a continuous time Markov chain called credit migration process studied by Bielecki and Rutkowski in [4]. Hence, our copula, which depends on the credit ratings, will affect the dynamic of the default intensity. In fact, we define default intensity process by a Cox-Ingersoll-Ross (CIR) model whose parameters values depend on this copula.

The Cox-Ingersoll-Ross model was first considered to model the term structure of interest rate by Cox and al. in [7]. The study of this class of processes was caution by the fact that it allows us a closed form expression of Laplace transform (see Duffie and al. [8]) and models well the default intensity (Alfonsi and Brigo [1]). Moreover, Choi in [5] shown that regime switching CIR process captures more short term interest rate than standard models. In a econometric point of view, regime switching model were introduced by Hamilton in [16].

In this framework, we obtain explicit formulas to evaluate defaultable bond prices. More precisely, we obtain two different formulas to evaluate the Laplace transform of defaultable intensity. Firstly, we use the semi-affine property of the regime switching CIR model and then solve a system of Riccati's equations. Secondly, we extend the analytic approximation found in Choi and Wirjanto [6]. Indeed, the authors in [6] give an analytic approximation of the value of bond price with constant CIR parameters and with constant time step grid discretization of the model. We extend this result in three ways: firstly to evaluate conditional Laplace transform of a regime switching Cox Ingersoll Ross, secondly to evaluate defaultable regime switching bond price and thirdly in the case of non uniform deterministic time step model discretization (in our case, the time step model discretization depends on the regime switching stopping time). Then, we will apply these two formulas to price defaultable bond. We will illustrate the efficiency of our new modelization of regime switching intensity firstly by comparing the computing time of each formulas, secondly by showing (using real historical data based on the Greece spread CDS) that our model estimates well data and that each regime captures well some market features or economics behaviors.

The paper is structured as follow, in Section 2, we introduce the Markov copula, the credit migration process and the regime switching Cox-Ingersoll-Ross model. In Section 3, we give the two formulas to evaluate the conditional Laplace transform in this framework. Finally, in Section 4, we show some simulations to compare results given by each formulas and to illustrate the model and then we give some estimations on real data. 


\section{The defaultable model}

2.1. Credit migration model. Let $T>0$ be a fixed maturity time and denote by $\left(\Omega, \overline{\mathbb{F}}:=\left(\overline{\mathcal{F}}_{t}\right)_{[0, T]}, \mathbb{P}\right)$ an underlying probability space.

Definition 2.1. A notation is a label given by an entity which measures the viability of a firm. This graduate notation goes from 1 to K. 1 for the best economic or financial situation and $\mathrm{K}$ for the worst. We will call an indicator of notation a continuous time homogeneous Markov chain on the finite space $\mathcal{S}=\{1, \ldots, K\}$.

Let $A$ and $B$ be two firms with their own indicator of notation $\left(X^{A}\right)_{t \in[0, T]}$ and $\left(X^{B}\right)_{t \in[0, T]}$. Hence $X^{A}$ and $X^{B}$ are two Markov chains with generator matrix $\Pi^{A}$ and $\Pi^{B}$. We recall that the generator matrix of $C \in\{A, B\}$ is given by $\Pi_{i j}^{C} \geq 0$ if $i \neq j$ for all $i, j \in \mathcal{S}$ and $\Pi_{i i}^{C}=-\sum_{j \neq i} \Pi_{i j}$ otherwise. We can remark that $\Pi_{i j}^{C}$ represents the intensity of the jump from state $i$ to state $j$. Moreover, we denote by $\mathcal{F}_{t}^{A}:=\left\{\sigma\left(X_{s}^{A}\right) ; 0 \leq s \leq t\right\}$ and $\mathcal{F}_{t}^{B}:=\left\{\sigma\left(X_{s}^{B}\right) ; 0 \leq s \leq t\right\}$ the natural filtrations generated by $X^{A}$ and $X^{B}$.

2.1.1. Markov Copula. Let denote by $X$ the bivariate process $X=\left(X^{A}, X^{B}\right)$, which is a finite continuous time Markov chain with respect to its natural filtration $\mathcal{F}^{X}=\mathcal{F}^{A, B}$. We recall now the Corollary 5.1 of Bielecki and al. [2], applied to our case, which gives the condition that the components of the bivariate processes $X$ are themselves Markov chain with respect to their own natural filtration.

Corollary 2.2. Consider two Markov chains $X^{A}$ and $X^{B}$, with respect to their own filtrations $\mathcal{F}^{A}$ and $\mathcal{F}^{B}$, and with values in $\mathcal{S}$. Suppose that their respective generators are $\Pi_{i j}^{A}$ and $\Pi_{h k}^{B}$ with $i, j, h$ and $k$ are in $\mathcal{S}$. Consider the system of equations in the unknown $\Pi_{i j, h k}^{X}$ where $i, j, h, k \in \mathcal{S}$ and $(i, h) \neq(j, k)$ :

$$
\begin{aligned}
& \sum_{k \in \mathcal{S}} \Pi_{i j, h k}^{X}=\Pi_{i j}^{A}, \quad \forall h, i, j \in \mathcal{S}, i \neq j, \\
& \sum_{j \in \mathcal{S}} \Pi_{i j, h k}^{X}=\Pi_{h k}^{B}, \quad \forall i, h, k \in \mathcal{S}, h \neq k .
\end{aligned}
$$

Suppose that the above system admits a solution such that the matrix $\Pi^{Z}:=$ $\left(\Pi_{i j, h k}^{Z}\right)_{i, j, h, k \in \mathcal{S}}$ with

$$
\Pi_{i i, h h}^{X}=-\sum_{(j, k) \in \mathcal{S} \times \mathcal{S},(j, k) \neq(i, h)} \Pi_{i j, h k}^{X}
$$

properly defines an infinitesimal generator of a Markov chain with values in $\mathcal{S} \times \mathcal{S}$. Consider, the bivariate Markov chain $X=\left(X^{A}, X^{B}\right)$ on $\mathcal{S} \times \mathcal{S}$ with generator matrix $\Pi^{X}$. Then, the components $X^{A}$ and $X^{B}$ are Markov chains with respect to their own filtrations, their generators are $\Pi^{A}$ and $\Pi^{B}$. 
Definition 2.3. A Markov copula between the Markov chains $X^{A}$ and $X^{B}$ is any solution to system (1) such that the matrix $\Pi^{X}$, with $\Pi_{i i, h}^{X}$ given in (2), properly defines an infinitesimal generator of a Markov chain with values in $\mathcal{S} \times \mathcal{S}$.

We get also that the infinitesimal generator process of $X$ can be written as

$$
\Pi^{X}=\left(\begin{array}{ccc}
\pi_{(1,1)} & \ldots & \pi_{(1, K)} \\
\pi_{(2,1} & \ldots & \pi_{(2, K)} \\
\vdots & & \vdots \\
\pi_{(K, 1)} & \ldots & \pi_{(K, K)}
\end{array}\right)
$$

Then the possible states are $N:=K^{2}$ couples which are given by

$$
\mathcal{E}:=\{(1,1),(1,2), \ldots,(1, K),(2,1), \ldots,(2, K), \ldots(K, 1), \ldots,(K, K)\} .
$$

2.1.2. Markov copula in the hazard rate framework. We denote by $\mathbb{F}:=\left(\mathcal{F}_{t}\right)_{t \in[0, T]}$ the filtration such that $\mathcal{F}_{t}=\overline{\mathcal{F}}_{t} \vee \mathcal{F}_{t}^{X}$. Let $\tau^{A}$ and $\tau^{B}$ be the two default times of firms A and B. Let define for all $t \in[0, T], H_{t}^{A}=1_{\left\{\tau^{A} \leq t\right\}}$ and $H_{t}^{B}=1_{\left\{\tau^{B} \leq t\right\}}$. We define now some others filtrations

$$
\mathcal{G}_{t}^{A}=\mathcal{F}_{t} \vee \mathcal{H}_{t}^{B}, \quad, \quad \mathcal{G}_{t}^{B}=\mathcal{F}_{t} \vee \mathcal{H}_{t}^{A} \quad \text { and } \quad \mathcal{G}_{t}=\mathcal{F}_{t} \vee \mathcal{H}_{t}^{A} \vee \mathcal{H}_{t}^{B},
$$

where $\mathcal{H}^{A}$ (resp. $\mathcal{H}^{B}$ ) is the natural filtration generated by $H^{A}$ (resp. $H^{B}$ ) and we will denote $\mathbb{G}:=\left(\mathcal{G}_{t}\right)_{t \in[0, T]}, \mathbb{G}^{A}:=\left(\mathcal{G}_{t}^{A}\right)_{t \in[0, T]}$ and $\mathbb{G}^{B}:=\left(\mathcal{G}_{t}^{B}\right)_{t \in[0, T]}$. Let now consider $\lambda^{i}:=\lambda^{i}(X)$, for $i \in\{A, B\}$ two $\mathbb{F}$-progressively non-negative processes defined on $(\Omega, \mathcal{G}, \mathbb{P})$ endowed with the filtration $\mathbb{F}$. We assume that $\int_{0}^{\infty} \lambda^{i}\left(X_{s}\right) d s=+\infty$ and we set:

$$
\tau^{i}=\inf \left\{t \in \mathbb{R}^{+}, \int_{0}^{t} \lambda^{i}\left(X_{s}\right) d s \geq-\ln \left(U^{i}\right)\right\}, \quad i \in\{A, B\} .
$$

where $U^{i}$ are mutually independent uniform random variables defined on $(\Omega, \mathcal{G}, \mathbb{P})$ which are independent of $\lambda^{i}$. The stopping times $\tau^{A}$ and $\tau^{B}$ are totally inaccessible and conditionally independent given the filtration $\mathbb{F}$, moreover the $(\mathcal{H})$ hypothesis is satisfied (i.e. that every local $\mathbb{F}$-martingale is a local $\mathbb{G}$-martingale too). The process $\lambda^{i}$ is called the $\mathbb{F}$-intensity of the firm $i$ and we have that $M_{t}^{i}=H_{t}^{i}-\int_{0}^{t \wedge \tau^{i}} \lambda^{i}\left(X_{s}\right) d s$ are $\mathbb{G}$-martingales. In general case, processes $\lambda^{i}$ are $\mathbb{F} \vee \mathbb{G}^{(i)}$-adapted which jump when any default occurs. This jump impacts the default of the firm and makes some correlation between the firms. In our case, the correlation is constructed using the $\mathbb{F}$-Markov chain $X=\left(X^{A}, X^{B}\right)$. Since from the explicit formula of the intensity given the survey probability for each $i \in\{A, B\}$ :

$$
\lambda_{t}^{i}=-\left.\frac{1}{\mathbb{P}\left(\tau^{i} \geq t \mid \mathcal{G}_{t}^{i}\right)} \frac{d \mathbb{P}\left(\tau^{i} \geq \theta \mid \mathcal{G}_{t}^{i}\right)}{d \theta}\right|_{\theta=t},
$$


we can find, from Bielecki and al. [3] (Example 4.5.1 p 94), that the formula of the conditional survey probability is given by:

$$
\mathbb{P}\left(\tau^{i} \geq \theta \mid \mathcal{G}_{t}\right)=1_{\left\{\tau^{i} \geq t\right\}} \mathbb{E}\left[e^{-\int_{t}^{\theta} \lambda^{i}\left(X_{s}\right) d s} \mid \mathcal{F}_{t}\right],
$$

for $i \in\{A, B\}$. The Markov chain $X$ will explain how the curve of the default bond moves with different states (regimes) of the financial market.

2.1.3. Construction of the Markov chain. We are now going to present the canonical construction of a conditional Markov chain X, based on a given filtration $\mathbb{F}$ and a stochastic infinitesimal generator $\Pi^{X}$. This construction can be found in Bielecki and Rukowski [4] or Eberlein and Ozkan [10], which we follow closely in the exposition. Each component $\Pi_{i j}^{X}: \Omega \times[0, T] \rightarrow \mathbb{R}^{+}$are bounded, $\mathbb{F}$ progressively measurable stochastic processes. The process $\mathrm{X}$ is constructed from an initial distribution $\mu$ and the $\overline{\mathbb{F}}$-conditional adapted infinitesimal generator $\Pi^{X}$ by enlarging the underlying probability space $\left(\Omega, \overline{\mathcal{F}}, \mathbb{P}_{T}\right)$ to a probability space denoted in the sequel by $\left(\Omega, \mathcal{F}, \mathbb{Q}_{T}\right)$. The new probability space is obtained as a product space of the underlying one with a probability space supporting the initial distribution $\mu$ of $\mathrm{X}$ and a probability space supporting a sequence of uniformly distributed random variables, which control, together with the entries of the infinitesimal generator $\Pi^{X}$, the laws of jump times $\left(\tau_{k}\right)_{k \in \mathbb{N}}$ of $\mathrm{X}$ and jump heights. Thus, we denote by $\mathbb{F}$ its trivial extension from the original probability space $\left(\Omega, \overline{\mathcal{F}}, \mathbb{P}_{T}\right)$ to $\left(\Omega, \mathcal{F}, \mathbb{Q}_{T}\right)$. However an important step of this construction is that they construct a discrete time process $\left(\bar{X}_{k}\right)_{k \in \mathbb{N}}$ which allows us to construct the credit migration process $X$ as $X_{t}:=\bar{X}_{k-1}$, for all $t \in$ $\left[\tau_{k-1}, \tau_{k}\left[, \quad k \geq 1\right.\right.$, where $\tau_{k}$ are the jump times. Moreover, an important result is that the progressive enlargement of filtration $\mathcal{F}_{t}:=\overline{\mathcal{F}}_{t} \vee \mathcal{F}_{t}^{X}, t \in[0, T]$ satisfies the $(\mathcal{H})$-hypothesis. In the sequel, we will work under the enlarging probability space $\left(\Omega, \mathcal{F}, \mathbb{Q}_{T}\right)$. The expectations will be taken under the probability measure $\mathbb{Q}_{T}$ but for simplicity of notation, we will write $\mathbb{E}$ for $\mathbb{E}^{\mathbb{Q}_{T}}$.

\subsection{Pricing defaultable bond with Markov copula.}

2.2.1. Defaultable Model. Let $W$ be a standard real Brownian motion with filtration $\overline{\mathcal{F}_{t}}=\sigma\left\{W_{s} ; 0 \leq s \leq t\right\}$. We recall that a Cox Ingersoll Ross (CIR) process is the solution of the stochastic differential equation given by $d \lambda_{t}=$ $\kappa\left(\theta-\lambda_{t}\right) d t+\sigma \sqrt{\lambda_{t}} d W_{t}, \quad t \in[0, T]$, where $\kappa, \theta$ and $\sigma$ are constants which satisfy the condition $\sigma>0$ and $\kappa \theta>0$. We will assume that $\lambda_{0} \in \mathbb{R}^{+}$and that $2 \kappa \theta \geq \sigma^{2}$. This is to ensure that the process $\left(\lambda_{t}\right)$ is positive. We will now define the notion of regime switching CIR.

Definition 2.4. Let $(X)_{t}$ be a two-dimensional continuous time Markov chain on finite space $\mathcal{S}^{2}:=\{1, \ldots, K\}^{2}$. We will call a Regime switching CIR the process $\left(\lambda_{t}\right)$ which is the solution of the stochastic differential equation given by

$$
d \lambda_{t}=\kappa\left(X_{t}\right)\left(\theta\left(X_{t}\right)-\lambda_{t}\right) d t+\sigma\left(X_{t}\right) \sqrt{\lambda_{t}} d W_{t}, \quad t \in[0, T] .
$$

For all $j \in\{1, \ldots, K\}^{2}$, we have that $\kappa(j) \theta(j)>0$ and $2 \kappa(j) \theta(j) \geq \sigma(j)^{2}$. 
For simplicity, we will denote the values $\kappa\left(X_{t}\right), \theta\left(X_{t}\right)$ and $\sigma\left(X_{t}\right)$ by $\kappa_{t}, \theta_{t}$ and $\sigma_{t}$.

Assumption 1. We assume that both intensities processes $\lambda^{A}$ and $\lambda^{B}$ defined in Section 2.1.2 follow a regime switching $C I R$ given for $i=\{A, B\}$ by $d \lambda_{t}^{i}=\kappa\left(X_{t}\right)\left(\theta\left(X_{t}\right)-\lambda_{t}^{i}\right) d t+\sigma\left(X_{t}\right) \sqrt{\lambda_{t}^{i}} d W_{t}$, for all $t \in[0, T]$.

Remark 2.1. We have that the intensity process $\left(\lambda_{t}^{i}\right)$ depends on the value of the credit migration process $X=\left(X^{A}, X^{B}\right)$. Hence each firm A and $\mathrm{B}$ has an increasing sequence of $\mathbb{F}^{X}$-stopping times given by:

- for the firm $\mathrm{A}$ it is $0 \leq \tau_{1}^{A}<\tau_{2}^{A}<\cdots<\tau_{n}^{A} \leq T$.

- for the firm B it is $0 \leq \tau_{1}^{B}<\tau_{2}^{B}<\cdots<\tau_{m}^{B} \leq T$.

Hence with these two sequences, we can construct another sequence by a rearrangement of these two sequences in one. Indeed, we can put every stopping time $\tau_{i}^{A}, i \in\{1, \ldots n\}$ and $\tau_{j}^{B}, j \in\{1, \ldots, m\}$ in an increasing order. We obtain a new increasing sequence of stopping times of size $M \in \mathbb{N}$ given by $0 \leq \tau_{1}<\tau_{2}<\cdots<\tau_{M} \leq T$. As an example of this construction:

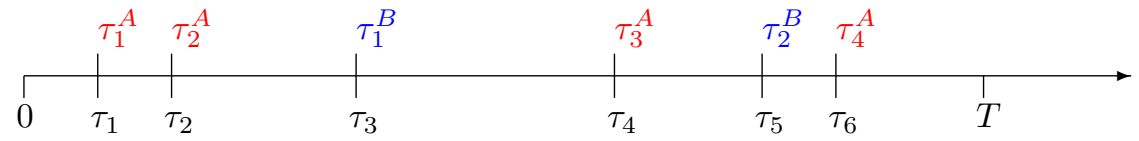

Remark 2.2. With this construction, we have that on each time interval $t \in$ $\left[\tau_{k}, \tau_{k+1}\right.$ [ that the regime switching CIR process $\lambda^{i}$ defined in Assumption 1 is a classical CIR with constant parameters.

2.2.2. Zero coupon bond price. We can now define the defaultable Zero coupon bond price.

Definition 2.5. We will denote by $\left(D_{t, T}^{i}\right)_{t \in[0, T]}, i=\{A, B\}$ the price of a defaultable discounted bond price which pays $\$ 1$ at the maturity $\mathrm{T}$.

Using the partitioning time, the notation defined in the previous subsection and the general asset pricing theory in Harrison and Pliska [17] and [18], the conditional defaultable discounted bond price $D_{t, T}$ is given by

Proposition 2.6. For $i=\{A, B\}$, we have for all $t \in[0, T]$ that

$$
D_{t, T}^{i}=\left(1-H_{t}^{i}\right) \mathbb{E}\left[\exp \left(-\int_{t}^{T}\left(r_{s}+\lambda_{s}^{i}\right) d s\right) \mid \mathcal{F}_{T}^{X}, \lambda_{t}\right]
$$

Remark 2.3. (1) The quantity $\left(r_{t}+\lambda_{t}^{i}\right)_{t \in[0, T]}$ can be seen as a default-adjusted interest rate process. The part $\left(\lambda_{t}^{i}\right)_{t \in[0, T]}$ is the risk-neutral mean loss rate of the instrument due to the default of the firm $i \in\{A, B\}$. The quantity $\left(r_{t}+\lambda_{t}^{i}\right)_{t \in[0, T]}$ therefore represents the probability and the timing of default, as well as for the effect of losses on default. This model allows us to capture an economic health of each firm since for each firm $i \in\{A, B\}$, the stochastic process $\left(\lambda_{t}^{i}\right)$ has parameters whose values depend on the credit notation of the firms. And 
by the construction of the migration process $X$, we have also correlation between each firm notation. This allows the model to capture financial health correlation between each firm, like the impact of the default of one firm against the others. (2) In the sequel we will assume that we know the full history of the Markov chain $X$.

Our aim is so to obtain explicit formulas of (5) when the intensities processes depend on a Markov copula. This is done by the following Theorem using two different methods to evaluate the conditional Laplace transform of $\lambda^{i}$. The first one uses a Ricatti approach and the second one an analytical approximation.

Theorem 2.7. Under Assumptions 1 and assuming that $X$ is independent of $W$ and that the risk-free interest rate $r$ is a deterministic function, we have for $i \in\{A, B\}$ that the defaultable bond price can be obtained by two formulas:

(1) Riccati Approach:

$$
\begin{aligned}
& D_{0, T}^{i}=\mathbb{E}\left[\exp \left(-\int_{0}^{T} r_{s} d s\right) \exp \left\{-\sum_{j=1}^{M} B_{M-j}\left(\Delta_{t_{j-1}}\right)\right\} \exp \left(-A_{0}\left(\Delta_{t_{0}}, i_{0}\right) \lambda\right)\right] \\
& \text { where } \\
& A_{0}\left(\Delta_{t_{0}}\right)= \frac{2}{\gamma^{1}+\kappa^{1}}-\frac{4 \gamma^{1}}{\gamma^{1}+\kappa^{1}} \frac{1}{\left(\gamma^{1}+\kappa^{1}\right) \exp \left(\gamma^{1} \Delta_{t_{0}}\right)+\gamma^{1}-\kappa^{1}}, \\
& B_{M-j}\left(\Delta_{t_{j-1}}\right)=-\frac{\kappa^{M-j+1} \theta^{M-j+1}\left(\gamma^{M-j+1}+\kappa^{M-j+1}\right)}{\left(\sigma^{M-j+1}\right)^{2}} \Delta_{t_{j-1}} \\
&+2 \frac{\kappa^{M-j+1} \theta^{M-j+1}}{\left(\sigma^{M-j+1}\right)^{2}}\left[-\ln \left(2 \gamma^{M-j+1}\right)\right. \\
&\left.+\ln \left(\left(\gamma^{M-j+1}+\kappa^{M-j+1}\right) \exp \left(\gamma^{M-j+1} \Delta_{t_{j-1}}\right)+\gamma^{M-j+1}-\kappa^{M-j+1}\right)\right],
\end{aligned}
$$

and

$$
\gamma^{M-j+1}=\sqrt{\left(\kappa^{M-j+1}\right)^{2}+2\left(\sigma^{M-j+1}\right)^{2}},
$$

where we denote for simplicity $\kappa^{j}=\kappa\left(X_{t_{j}}\right), \theta^{j}=\theta\left(X_{t_{j}}\right)$ and $\sigma^{j}=$ $\sigma\left(X_{t_{j}}\right)$

(2) Analytic Approximation:

$D_{0, T}^{i}=\mathbb{E}\left[\exp \left(-\int_{0}^{T} r_{s} d s\right) \exp \left\{\sum_{k=1}^{n}-\frac{u}{2} C^{1}(k)+\ln \left(\mathbb{E}_{\lambda_{0}, X}^{t_{0}}\left[C^{2}(k)\right]\right)\right\}\right]$,

where the sequence $a$ is given by $a_{n-1}=1+\frac{h_{n}}{h_{n-1}}+\frac{h_{n}}{h_{n-1}} a_{n}\left(1-h_{n} \kappa_{n-1}\right)$, $a_{n}=1$ and $C^{1}, C^{2}$ are given by

$C^{1}(k):=h_{n-k+1}^{2} a_{n-k+1} \kappa_{n-k} \theta_{n-k}-\frac{u}{2} h_{1} \lambda_{0}\left[1+a_{1}\left(1-\kappa_{0} h_{1}\right)\right]$,

$C^{2}(k):=\exp \left(\frac{h_{n-k+1}^{3}}{8} u^{2} \sigma_{n-k}^{2} a_{n-k+1}^{2}\left[\lambda_{0}+\sum_{i=0}^{n-k} \kappa_{i}\left(\theta_{i}-\lambda_{i}\right) h_{i+1}+\sum_{i=0}^{n-k} \sigma_{i} \sqrt{\lambda_{i}} \Delta W_{i}\right]\right)$. 
Remark 2.4. The hypothesis that $X$ is independent of $W$ has an economic sense since for example $X=\left(X^{A}, X^{B}\right)$ could represent the credit notation of two countries given by an exogenous entity like a credit rating agencies.

Proof. The proof will be given in the sequel following the Section 3.

\section{Conditional Laplace transform formulas}

We are now going to prove the Theorem 2.7. More precisely, we will find two explicit formulas to evaluate the conditional Laplace transform of $\lambda$ with respect to $\mathrm{X}$ denoted by $\Phi$. It is given, for all $u \in \mathbb{C}$, by

$$
\Phi_{0, T, \lambda, X}(u)=\mathbb{E}\left[\exp \left(-u \int_{0}^{T} \lambda_{s} d s\right) \mid \lambda_{0}=\lambda, \mathcal{F}_{T}^{X}\right]=\mathbb{E}_{\lambda, X}\left[\exp \left(-u \int_{0}^{T} \lambda_{s} d s\right)\right] .
$$

Hence, our defaultable bond price formulas will be obtained as a particular case of this equation by taking $u=1$.

3.1. A Ricatti approach. By Remark 2.1, there exists an increasing sequence of $\mathbb{F}^{X}$-stopping times in interval $[0, T]$, where the value of the Markov chain changes. We denote by $\Gamma$ this subdivision $0=\tau_{0}<\tau_{1}<\cdots<\tau_{M}=T$. So in each time interval $\left[\tau_{k}, \tau_{k+1}[, k \in\{1, \ldots n\}, X\right.$ is constant and so the CIR regime switching process $\lambda$ has constant parameters.

Proposition 3.1. The conditional Laplace transform of the regime switching CIR process (for $u=1$ ) between time $\left[\tau_{k}, \tau_{k+1}\left[\right.\right.$ with $\lambda_{\tau_{k}}=\lambda$ and $X_{\tau_{k+1}}=j \in \mathcal{S}^{d}$ is given by

$$
\begin{aligned}
\Phi_{\tau_{k}, \tau_{k+1}, j} & :=\mathbb{E}\left[\exp \left(-\int_{\tau_{k}}^{\tau_{k+1}} \lambda_{s} d s\right) \mid \lambda_{\tau_{k}}=\lambda, X_{\tau_{k+1}}=j\right], \\
& =\exp \left\{-A\left(\Delta_{t_{k}}, j\right) \lambda-B\left(\Delta_{t_{k}}, j\right)\right\}
\end{aligned}
$$

where $\Delta_{t_{k}}=\tau_{k+1}-\tau_{k}$ and

$$
\begin{gathered}
A\left(\Delta_{t_{k}}, j\right)=\frac{2}{\gamma_{j}+\kappa_{j}}-\frac{4 \gamma_{j}}{\gamma_{j}+\kappa_{j}} \frac{1}{\left(\gamma_{j}+\kappa_{j}\right) \exp \left(\gamma_{j} \Delta_{t_{k}}\right)+\gamma_{j}-\kappa_{j}}, \\
B\left(\Delta_{t_{k}}, j\right)=-\frac{\kappa_{j} \theta_{j}\left(\gamma_{j}+\kappa_{j}\right)}{\sigma_{j}^{2}} \Delta_{t_{k}}+2 \frac{\kappa_{j} \theta_{j}}{\sigma_{j}^{2}} \ln \left(\left(\gamma_{j}+\kappa_{j}\right) \exp \left(\gamma_{j} \Delta_{t_{k}}\right)+\gamma_{j}-\kappa_{j}\right) \\
-2 \frac{\kappa_{j} \theta_{j}}{\sigma_{j}^{2}} \ln \left(2 \gamma_{j}\right), \\
\gamma_{j}=\sqrt{\kappa_{j}^{2}+2 \sigma_{j}^{2}} .
\end{gathered}
$$

Proof. We recall that the constant parameter CIR process is an affine process (see Duffie and al. [8]). So as in each step of time $\left[\tau_{k}, \tau_{k+1}[\right.$, the stochastic process $\mathrm{X}$ is constant. So the process $\lambda$ is a classical CIR with constant parameters on each step. So on each time interval $\left[\tau_{k}, \tau_{k+1}[\right.$, the process $\lambda$ is affine, hence we can assume that the expression of $\Phi_{\tau_{k}, \tau_{k+1}, j}$ is given by the form 
$\exp \left\{-A\left(\Delta_{t_{k}}, j\right) \lambda_{\tau_{k}}-B\left(\Delta_{t_{k}}, j\right)\right\}$ for some functions $A\left(\Delta_{t_{k}}, j\right)$ and $B\left(\Delta_{t_{k}}, j\right)$ solution of a system of Riccati equation. Then the expected result is well known and can be found for instance in Cox and al. [7].

We would like now to give an explicit form of the conditional Laplace transform of the CIR process between time 0 and T. This is done by the following Theorem.

Theorem 3.2. Assume that the intensity process $\left(\lambda_{t}\right)$ follows a regime switching $C I R$, then we have for all $\lambda_{0}=\lambda>0$ and $X_{\tau_{1}}=i_{0} \in \mathcal{S}^{d}$ that

$$
\begin{aligned}
\Phi_{0, T, \lambda, X}(1) & =\mathbb{E}\left[\exp \left(-\int_{0}^{T} \lambda_{s} d s\right) \mid \lambda_{0}=\lambda, \mathcal{F}_{T}^{X}\right]:=\mathbb{E}_{\lambda, X}\left[\exp \left(-\int_{0}^{T} \lambda_{s} d s\right)\right], \\
& =\exp \left\{-\sum_{j=1}^{M} B_{M-j}\left(\Delta_{t_{j-1}}\right)\right\} \exp \left(-A_{0}\left(\Delta_{t_{0}}, i_{0}\right) \lambda\right) .
\end{aligned}
$$

where $A_{0}\left(\Delta_{t_{0}}\right), B_{M-j}\left(\Delta_{t_{j-1}}\right)$ and $\gamma^{M-j+1}$ are defined in Theorem 2.7 (see (7), (8) and (9)).

Proof. We have a sequence of increasing times $0=\tau_{0}<\tau_{1}<\cdots<\tau_{M}=T$ where the Markov chain $X$ changes its value. Hence

$$
\mathbb{E}_{\lambda, X}\left[\exp \left(-\int_{0}^{T} \lambda_{s} d s\right)\right]=\mathbb{E}_{\lambda, X}\left[\prod_{k=0}^{M-1} \exp \left(-\int_{\tau_{k}}^{\tau_{k+1}} \lambda_{s} d s\right)\right] .
$$

By hypothesis, $X$ is independent of $W$, then conditioning with respect to $\overline{\mathcal{F}}_{\tau_{M-1}}$ $:=\overline{\mathcal{F}}_{M-1}$, we obtain

$$
\begin{aligned}
& \mathbb{E}_{\lambda, X}\left[\exp \left(-\int_{0}^{T} \lambda_{s} d s\right)\right]=\mathbb{E}_{\lambda, X}\left[\mathbb{E}\left[\prod_{k=0}^{M-1} \exp \left(-\int_{\tau_{k}}^{\tau_{k+1}} \lambda_{s} d s\right) \mid \overline{\mathcal{F}}_{M-1}\right]\right], \\
= & \mathbb{E}_{\lambda, X}\left[\prod_{k=0}^{M-2} \exp \left(-\int_{\tau_{k}}^{\tau_{k+1}} \lambda_{s} d s\right) \mathbb{E}\left[\exp \left(-\int_{\tau_{M-1}}^{\tau_{M}} \lambda_{s} d s\right) \mid \overline{\mathcal{F}}_{M-1}\right]\right] .
\end{aligned}
$$

Moreover, we know that $\mathbb{E}\left[\exp \left(-\int_{\tau_{M-1}}^{\tau_{M}} \lambda_{s} d s\right) \mid \overline{\mathcal{F}}_{M-1}\right]$ is equal to

$\Phi\left(\tau_{M-1}, \tau_{M}, X_{M}\right)$, where $X_{M}$ means $X_{\tau_{M}}$. So applying Proposition 3.1, we get

$$
\begin{aligned}
\mathbb{E}\left[\exp \left(-\int_{\tau_{M-1}}^{\tau_{M}} \lambda_{s} d s\right) \mid \overline{\mathcal{F}}_{M-1}\right]= & \exp \left\{-A_{M-1}\left(\Delta_{t_{M-1}}, X_{M}\right) \lambda_{\tau_{M-1}}\right. \\
& \left.-B_{M-1}\left(\Delta_{t_{M-1}}, X_{M}\right)\right\}
\end{aligned}
$$

We recall that the quantities $A_{M-1}\left(\Delta_{t_{M-1}}, X_{M}\right)$ and $B_{M-1}\left(\Delta_{t_{M-1}}, X_{M}\right)$ are constants. To simplify the notation of the calculus we will denote by $A_{k-1}$ (resp. $\left.B_{k-1}\right)$ the quantity $A_{k-1}\left(\Delta_{t_{k-1}}, X_{k}\right)$ (resp. $B_{k-1}\left(\Delta_{t_{k-1}}, X_{k}\right)$ ). Hence 
replacing this result in the expectation (16) gives

$$
\begin{aligned}
\mathbb{E}_{\lambda, X}\left[\exp \left(-\int_{0}^{T} \lambda_{s} d s\right)\right] & =\mathbb{E}_{\lambda, X}\left[\prod_{k=0}^{M-2} \exp \left(-\int_{\tau_{k}}^{\tau_{k+1}} \lambda_{s} d s\right) \exp \left\{-A_{M-1} \lambda_{\tau_{M-1}}-B_{M-1}\right\}\right], \\
& =\exp \left\{-B_{M-1}\right\} \mathbb{E}_{\lambda, X}\left[\prod_{k=0}^{M-2} \exp \left(-\int_{\tau_{k}}^{\tau_{k+1}} \lambda_{s} d s-A_{M-1} \lambda_{\tau_{M-1}}\right)\right] .
\end{aligned}
$$

We condition again the next quantity with respect to $\overline{\mathcal{F}}_{M-2}$ to obtain

$$
\begin{aligned}
\mathbb{E}_{\lambda, X}\left[\exp \left(-\int_{0}^{T} \lambda_{s} d s\right)\right]= & \exp \left\{-B_{M-1}\right\} \mathbb{E}_{\lambda, X}\left[\prod_{k=0}^{M-3} \exp \left(-\int_{\tau_{k}}^{\tau_{k+1}} \lambda_{s} d s\right)\right. \\
& \left.\mathbb{E}\left[\exp \left(-\int_{\tau_{M-2}}^{\tau_{M-1}} \lambda_{s} d s-A_{M-1} \lambda_{\tau_{M-1}}\right) \mid \overline{\mathcal{F}}_{M-2}\right]\right] .
\end{aligned}
$$

To continue, we need to evaluate the conditional expectation:

$$
\varphi_{\tau_{M-2}, \Delta_{t_{M-2}}}:=\mathbb{E}\left[\exp \left(-\int_{\tau_{M-2}}^{\tau_{M-1}} \lambda_{s} d s-A_{M-1} \lambda_{\tau_{M-1}}\right) \mid \overline{\mathcal{F}}_{M-2}\right] .
$$

Lemma 3.3. Assume for all $k \in\{1, \ldots, M\}$ that the conditional expectation $\varphi_{\tau_{M-k}, \Delta_{t_{M-k}}}$ has an exponential affine structure form given by

$\varphi_{\tau_{M-k}, \Delta_{t_{M-k}}}=\exp \left(-A_{M-k}\left(\Delta_{t_{M-k}}, X_{M-k+1}\right) \lambda_{\tau_{M-k}}-B_{M-k}\left(\Delta_{t_{M-k}}, X_{M-k+1}\right)\right)$.

Then we can find explicit forms for functions $A_{M-k}\left(\Delta_{t_{M-k}}, X_{M-k+1}\right)$ and $B_{M-k}\left(\Delta_{t_{M-k}}, X_{M-k+1}\right)$ which are given explicitly by equations (13) and (14) under the conditions that $A_{M-k}(0)=A_{M-k+1}$ and $B_{M-k}(0)=0$.

Proof. Let

$$
\varphi_{\tau_{M-k}, \Delta_{t_{M-k}}}:=\mathbb{E}\left[\exp \left(-\int_{\tau_{M-k}}^{\tau_{M-k+1}} \lambda_{s} d s-A_{M-k+1} \lambda_{\tau_{M-k+1}}\right) \mid \overline{\mathcal{F}}_{M-k}\right],
$$

then

$\varphi_{\tau_{M-k}, \Delta_{t_{M-k}}}=\mathbb{E}_{M-k}\left[\exp \left(-\int_{\tau_{M-k}}^{\tau_{M-k+1}} \lambda_{s} d s-A_{M-k+1} \lambda_{\tau_{M-k}+\Delta_{t_{M-k}}}\right)\right]$,

since $\Delta_{t_{M-k}}:=\tau_{M-k+1}-\tau_{M-k}$. Taking a small time interval $d t \ll \Delta_{t_{M-2}}$ to obtain that the previous quantity is equal to

$$
=\mathbb{E}_{M-k}\left[\mathbb{E}_{M-k+d t}\left[\exp \left(-\int_{\tau_{M-k}}^{\tau_{M-k+1}} \lambda_{s} d s-A_{M-k+1} \lambda_{\tau_{M-k}+\Delta_{t_{M-k}}}\right)\right]\right] .
$$

Thus the inside expectation is then equal to: $\mathbb{E}_{M-k}\left[\varphi\left(\tau_{M-k}+d t, \Delta_{t_{M-k}}-d t\right) \exp \left(-\int_{\tau_{M-k}}^{\tau_{M-k}+d t} \lambda_{s} d s\right)\right]$. We now use the hypothesis on the form of $\varphi$ and the simplified notations $A_{M-k}\left(\Delta_{t_{M-k}}-d t, X_{M-k+1}\right)$ $=A_{M-k}\left(\Delta_{t_{M-k}}-d t\right)$ and $B_{M-k}\left(\Delta_{t_{M-k}}-d t, X_{M-k+1}\right)=B_{M-k}\left(\Delta_{t_{M-k}}-d t\right)$, to get $\mathbb{E}_{M-k}\left[\exp \left(-\int_{\tau_{M-k}}^{\tau_{M-k}+d t} \lambda_{s} d s\right) \exp \left(-A_{M-k}\left(\Delta_{t_{M-k}}-d t\right) \lambda_{\tau_{M-k}+d t}\right)\right.$ 
$\left.\exp \left(-B_{M-k}\left(\Delta_{t_{M-k}}-d t\right)\right)\right]$. Then, for small $d t$ and using the stochastic differential equation of $\lambda$, we get that it is equal to

$$
\begin{aligned}
= & \mathbb{E}_{M-k}\left[\operatorname { e x p } \left\{-B_{M-k}\left(\Delta_{t_{M-k}}-d t\right)-\lambda_{\tau_{M-k}} d t-A_{M-k}\left(\Delta_{t_{M-k}}-d t\right)\right.\right. \\
& \left.\left.\times\left[\lambda_{\tau_{M-k}}+\kappa^{M-k+1}\left(\theta^{M-k+1}-\lambda_{\tau_{M-k}}\right) d t+\sigma^{M-k+1} \sqrt{\lambda_{\tau_{M-k}}} d W_{t}\right]\right\}\right]
\end{aligned}
$$

where $\kappa^{M-k+1}=\kappa\left(X_{\tau_{M-k+1}}\right), \theta^{M-k+1}=\theta\left(X_{\tau_{M-k+1}}\right)$ and $\sigma^{M-k+1}=\delta\left(X_{\tau_{M-k+1}}\right)$. Finally we obtain

$$
\begin{aligned}
& =\exp \left(-A_{M-k}\left(\Delta_{t_{M-k}}-d t\right) \lambda_{\tau_{M-k}}-A_{M-k}\left(\Delta_{t_{M-k}}\right) \kappa^{M-k+1}\left(\theta^{M-k+1}-\lambda_{\tau_{M-k}}\right) d t\right) \\
& \times \exp \left(-B_{M-k}\left(\Delta_{t_{M-k}}-d t\right)-\lambda_{\tau_{M-k}} d t\right) \times \exp \left(\frac{1}{2} A_{M-k}^{2}\left(\Delta_{t_{M-k}}\right)\left(\sigma^{M-k+1}\right)^{2} \lambda_{\tau_{M-k}} d t\right) .
\end{aligned}
$$

By identifying with the assumed expression of $\varphi$ in (17), we get

$$
\left\{\begin{aligned}
A_{M-k}\left(\Delta_{t_{M-k}}\right)= & A_{M-k}\left(\Delta_{t_{M-k}}-d t\right)-A_{M-k}\left(\Delta_{t_{M-k}}\right) \kappa^{M-k+1} d t \\
& -\frac{1}{2} A_{M-k}^{2}\left(\Delta_{t_{M-k}}\right)\left(\sigma^{M-k+1}\right)^{2} d t+d t . \\
B_{M-k}\left(\Delta_{t_{M-k}}\right)= & B_{M-k}\left(\Delta_{t_{M-k}}-d t\right)+A_{M-k}\left(\Delta_{t_{M-k}}\right) \kappa^{M-k+1} \theta^{M-k+1} d t .
\end{aligned}\right.
$$

Taking $d t$ close to zero,

$$
\left\{\begin{array}{l}
\frac{\partial A_{M-k}\left(\Delta_{t_{M-k}}\right)}{\partial \Delta_{t_{M-k}}}=-A_{M-k}\left(\Delta_{t_{M-k}}\right) \kappa^{M-k+1}-\frac{1}{2} A_{M-k}^{2}\left(\Delta_{t_{M-k}}\right)\left(\sigma^{M-k+1}\right)^{2}+1 \\
\frac{\partial B_{M-k}\left(\Delta_{t_{M-k}}\right)}{\partial \Delta_{t_{M-k}}}=A_{M-k}\left(\Delta_{t_{M-k}}\right) \kappa^{M-k+1} \theta^{M-k+1} .
\end{array}\right.
$$

with conditions for $\Delta_{t_{M-k}} \equiv 0, A_{M-k}(0)=A_{M-k+1}$ and $B_{M-k}(0)=0$. Hence by Proposition 3.1, we know the explicit forms of $A_{M-k}\left(\Delta_{t_{M-k}}\right)$ and $B_{M-k}\left(\Delta_{t_{M-k}}\right)$ which are given by equations (13), (14) with the recursive condition that $A_{M-k}(0)=A_{M-k+1}$ and initial condition $B_{M-k}(0)=0$.

We continue the proof of the Theorem 3.2, by applying the Lemma 3.3 with $k=2$, we obtain

$$
\begin{aligned}
& \mathbb{E}\left[\exp \left(-\int_{\tau_{M-2}}^{\tau_{M-1}} \lambda_{s} d s-A_{M-1} \lambda_{\tau_{M-1}}\right) \mid \overline{\mathcal{F}}_{M-2}\right] \\
= & \exp \left(-A_{M-2}\left(\Delta_{t_{M-2}}\right) \lambda_{\tau_{M-2}}-B_{M-2}\left(\Delta_{t_{M-2}}\right)\right),
\end{aligned}
$$

with deterministic function $A_{M-2}\left(\Delta_{t_{M-2}}\right)$ and $B_{M-2}\left(\Delta_{t_{M-2}}\right)$. Hence, we get

$$
\begin{gathered}
\mathbb{E}_{\lambda, X}\left[\exp \left(-\int_{0}^{T} r_{s} d s\right)\right]=\exp \left\{-B_{M-1}\right\} \times \\
\mathbb{E}_{\lambda, X}\left[\prod_{k=0}^{M-3} \exp \left(-\int_{\tau_{k}}^{\tau_{k+1}} \lambda_{s} d s\right) \exp \left(-A_{M-2}\left(\Delta_{t_{M-2}}\right) \lambda_{t_{M-2}}-B_{M-2}\left(\Delta_{t_{M-2}}\right)\right)\right] \\
=\exp \left\{-B_{M-1}-B_{M-2}\right\} \mathbb{E}_{\lambda, X}\left[\prod_{k=0}^{M-3} \exp \left(-\int_{\tau_{k}}^{\tau_{k+1}} \lambda_{s} d s-A_{M-2}\left(\Delta_{t_{M-2}}\right) \lambda_{\tau_{M-2}}\right)\right] .
\end{gathered}
$$


By iterating the conditioning with respect to $\overline{\mathcal{F}}_{M-k}$, $\mathrm{k}$ going to 3 to $\mathrm{M}$ and applying the Lemma 3.3 we finally obtain

$$
\mathbb{E}_{\lambda, X}\left[\exp \left(-\int_{0}^{T} \lambda_{s} d s\right)\right]=\exp \left\{-\sum_{j=1}^{M} B_{M-j}\right\} \exp \left(-A_{0}\left(\Delta_{t_{0}}\right) \lambda_{\tau_{0}}\right),
$$

with by hypothesis $\lambda_{\tau_{0}}=\lambda$ and $A_{0}\left(\Delta_{t_{0}}\right)=A_{0}\left(\Delta_{t_{0}}, X_{\tau_{1}}\right)$ with $X_{\tau_{1}}=i_{0} \in$ $\mathcal{S}^{d}$.

We can obtain the general expression of the conditional Laplace transform of the regime switching CIR process using Theorem 3.2.

Corollary 3.4. For all $u \in \mathbb{C}$, we have that the conditional Laplace transform of the regime switching CIR process with $\lambda_{0}=\lambda$ and $X_{\tau_{1}}=i_{0} \in \mathcal{S}^{d}$ is given by

$$
\Phi_{0, T, \lambda, X}(u)=\exp \left\{-\sum_{j=1}^{M} \tilde{B}_{M-j}\left(\Delta_{t_{M-j}}\right)\right\} \exp \left(-\tilde{A}_{0}\left(\Delta_{t_{0}}, i_{0}\right) \lambda\right),
$$

where the functions $\tilde{B}_{M-j}$ for $j=\{1, \ldots, M\}$ and $\tilde{A}_{0}$ are given by equations (7) and (8) taking parameters $\tilde{\kappa}^{j}:=\kappa\left(\tilde{X}_{\tau_{j}}\right)=\kappa^{j}, \tilde{\theta}^{j}:=\theta\left(\tilde{X}_{\tau_{j}}\right)=u \theta^{j}$ and $\tilde{\sigma}^{j}:=\sigma\left(\tilde{X}_{\tau_{j}}\right)=\sqrt{u} \sigma^{j}$.

Proof. Since $\mathbb{E}\left[\exp \left(-u \int_{0}^{T} \lambda_{s} d s\right) \mid \lambda_{0}=\lambda, \mathcal{F}_{T}^{X}\right]=\mathbb{E}_{\lambda, X}\left[\exp \left(-\int_{0}^{T}\left(u \lambda_{s}\right) d s\right)\right]$. This is the conditional Laplace transform of a process $(u \lambda)_{t}$ which is still a CIR process with new parameters $\tilde{\kappa}_{t}=\kappa_{t}, \tilde{\theta}_{t}=u \theta_{t}$ and $\tilde{\sigma}_{t}=\sqrt{u} \sigma_{t}$, for all $t \in[0, T]$. Hence applying Theorem 3.2 with this set of parameters gives the expected result.

3.2. Analytic approximation. We give now a second way to evaluate the defaultable bond. In fact, we give now an analytical approximation to evaluate the conditional Laplace transform of a regime switching CIR.

3.2.1. Construction of the new times grid. Let $\Delta_{t}$ be a fixed time step, then starting in time 0 we partition the time interval $[0, T]$ in time steps of

- size $\Delta_{t}$ if there is no jump of the Markov process between time 0 to $\Delta_{t}$.

- size $\tau_{1}$ if there is the first jump of the Markov process at stopping time $\tau_{1}$ less than $\Delta_{t}$.

Hence we denote by $h_{1}$ the first time step of size $\Delta_{t}$ or $\tau_{1}$. Then we will proceed as the following: at time $t_{k}$, corresponding of the time after the step $h_{k}$, we construct the step $h_{k+1}$ of size

- $\Delta_{t}$ if there is no jump of the Markov process between time $t_{k}$ to $t_{k}+\Delta_{t}$.

- $\tau_{i}$ if there is the $i$ jumps of the Markov process at stopping time $\tau_{i}$ less than $t_{k}+\Delta_{t}$. 
As an example of this construction

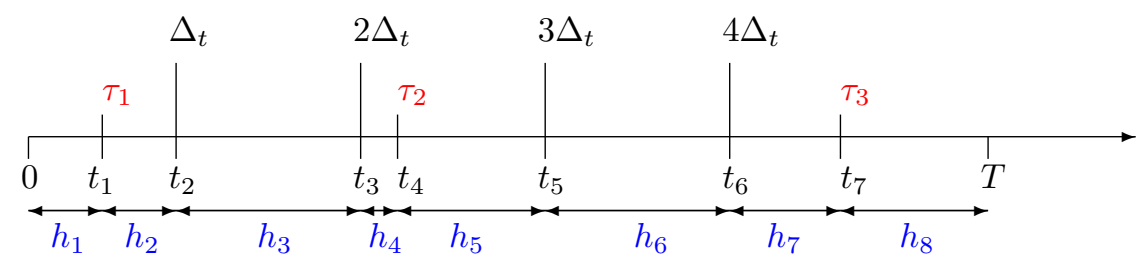

This construction implies that $h_{k}=t_{k}-t_{k-1} \leq \Delta_{t}$ and that the parameters of the regime switching CIR are constants (and bounded) in these each time intervals $\left[t_{k}, t_{k+1}[, k \in\{0,1, \ldots, n-1\}\right.$. It follows as an application of the tree property of conditional expectation that the conditional Laplace transform of $\lambda$ is given by

$$
\Phi_{0, T, \lambda, X}(u)=\mathbb{E}_{\lambda_{0}, X}^{t_{0}} \mathbb{E}_{\lambda, X}^{t_{1}} \ldots \mathbb{E}_{\lambda, X}^{t_{n-1}}\left[\exp \left(-u \int_{0}^{T} \lambda_{s} d s\right)\right] .
$$

Proposition 3.5. Let for all $k \in\{1, \ldots, n-1\}$,

$$
F_{k}=\exp \left(\frac{h_{n-k+1}^{3}}{8} u^{2} \sigma_{n-k}^{2} a_{n-k+1}^{2} \lambda_{n-k}\right) .
$$

Then, we have

$\mathbb{E}_{\lambda_{0}, X}^{t_{0}}\left[\frac{\exp \left(-u \int_{0}^{T} \lambda_{s} d s\right)}{\prod_{i=1}^{n-1} F_{i}}\right]=\exp \left(-\frac{u}{2} \sum_{k=1}^{n} h_{k}^{2} a_{k} \kappa_{k-1} \theta_{k-1}-\frac{u}{2} h_{1} \lambda_{0}\left[1+a_{1}\left(1-\kappa_{0} h_{1}\right)\right]\right) F_{n}$,

where $a_{n-1}=1+\frac{h_{n}}{h_{n-1}}+\frac{h_{n}}{h_{n-1}} a_{n}\left(1-h_{n} \kappa_{n-1}\right) \quad$ and $\quad a_{n}=1$.

Proof. Using trapezoidal rule, we obtain that the expectation at time $t_{n-1}$ is given by

$$
\begin{aligned}
\mathbb{E}_{\lambda_{0}, X}^{t_{n-1}}\left[\exp \left(-u \int_{0}^{T} \lambda_{s} d s\right)\right]= & \mathbb{E}_{\lambda_{0}, X}^{t_{n-1}}\left[\exp \left(-u \sum_{i=1}^{n}\left(\frac{\lambda_{i}+\lambda_{i-1}}{2} h_{i}\right)\right)\right] \\
= & \exp \left(-u \sum_{i=1}^{n-2}\left(\frac{\lambda_{i}+\lambda_{i-1}}{2} h_{i}\right)-u \frac{\lambda_{n-2}}{2} h_{n-1}\right) \\
& \times \mathbb{E}_{\lambda, X}^{t_{n-1}}\left[\exp \left(-\frac{u}{2}\left[h_{n} \lambda_{n}+h_{n} \lambda_{n-1}+h_{n-1} \lambda_{n-1}\right]\right)\right] .
\end{aligned}
$$

Using the approximation

$$
\lambda_{n} \simeq \lambda_{n-1}+\kappa_{n-1}\left(\theta_{n-1}-\lambda_{n-1}\right) h_{n}+\sigma_{n-1} \sqrt{\lambda_{n-1}} \Delta W_{n-1},
$$

where $\Delta W_{n-1}=W_{n}-W_{n-1}$ and denote by $G_{n-2}$ the quantity

$$
\exp \left(-u \sum_{i=1}^{n-2}\left(\frac{\lambda_{i}+\lambda_{i-1}}{2} h_{i}\right)-u \frac{\lambda_{n-2}}{2} h_{n-1}\right) .
$$


We obtain that $\mathbb{E}_{\lambda_{0}, X}^{t_{n-1}}\left[\exp \left(-u \int_{0}^{T} \lambda_{s} d s\right)\right]$ is equal to

$$
\begin{aligned}
& G_{n-2} \mathbb{E}_{\lambda_{0}, X}^{t_{n-1}}\left[\operatorname { e x p } \left(-\frac{u}{2}\left[h_{n}\left(\lambda_{n-1}+\kappa_{n-1}\left(\theta_{n-1}-\lambda_{n-1}\right) h_{n}+\sigma_{n-1} \sqrt{\lambda_{n-1}} \Delta W_{n-1}\right)\right.\right.\right. \\
& \left.\left.\left.+h_{n} \lambda_{n-1}+h_{n-1} \lambda_{n-1}\right]\right)\right], \\
& =G_{n-2} \exp \left(-\frac{u}{2}\left[h_{n} \lambda_{n-1}+h_{n}^{2} \kappa_{n-1} \theta_{n-1}-h_{n}^{2} \kappa_{n-1} \lambda_{n-1}+h_{n} \lambda_{n-1}+h_{n-1} \lambda_{n-1}\right]\right) \\
& \times \mathbb{E}_{\lambda_{0}, X}^{t_{n-1}}\left[\exp \left(-\frac{u}{2} h_{n} \sigma_{n-1} \sqrt{\lambda_{n-1}} \Delta W_{n-1}\right)\right] .
\end{aligned}
$$

Moreover we have that $\epsilon \sim \mathcal{N}(0,1)$ then for a constant $\mathrm{K}$ we know that

$$
\mathbb{E}[\exp (K \sqrt{T} \epsilon)]=\exp \left(\frac{K^{2} T}{2}\right) .
$$

Thus factorize by $-\frac{u \lambda_{n-1} h_{n-1}}{2}$, we obtain that $\mathbb{E}_{\lambda_{0}, X}^{t_{n-1}}\left[\exp \left(-u \int_{0}^{T} \lambda_{s} d s\right)\right]$ is equal to

$$
\begin{aligned}
& G_{n-2} \exp \left(\frac{u^{2}}{8} h_{n}^{3} \sigma_{n-1}^{2} \lambda_{n-1} a_{n}^{2}\right) \times \\
& \exp \left(-\frac{u \lambda_{n-1} h_{n-1}}{2}\left[1+\frac{h_{n}}{h_{n-1}}+\frac{h_{n}}{h_{n-1}} a_{n}\left(1-h_{n} \kappa_{n-1}\right)\right]-\frac{u}{2} h_{n}^{2} a_{n} \kappa_{n-1} \theta_{n-1}\right) \\
= & G_{n-2} \exp \left(-\frac{u}{2} h_{n}^{2} a_{n} \kappa_{n-1} \theta_{n-1}\right) \exp \left(-\frac{u \lambda_{n-1} h_{n-1}}{2} a_{n-1}\right) F_{1} .
\end{aligned}
$$

Hence

$\mathbb{E}_{\lambda_{0}, X}^{t_{n-1}}\left[\frac{\exp \left(-u \int_{0}^{T} \lambda_{s} d s\right)}{F_{1}}\right]=G_{n-2} \exp \left(\frac{u^{2}}{8} h_{n}^{3} \sigma_{n-1}^{2} \lambda_{n-1}\right) \exp \left(-\frac{u \lambda_{n-1} h_{n-1}}{2} a_{n-1}\right)$.

Then denoting $G_{n-3}=\exp \left(-u \sum_{i=1}^{n-3}\left(\frac{\lambda_{i}+\lambda_{i-1}}{2} h_{i}\right)-u \frac{\lambda_{n-3}}{2} h_{n-2}\right)$, we get the conditional expectation based on the information until $t_{n-2}$

$$
\begin{aligned}
& \mathbb{E}_{\lambda_{0}, X}^{t_{n-2}}\left[\mathbb{E}_{\lambda, X}^{t_{n-1}}\left[\frac{\exp \left(-u \int_{0}^{T} \lambda_{s} d s\right)}{F_{1}}\right]\right]=G_{n-3} \exp \left(-\frac{u}{2} h_{n}^{2} a_{n}^{2} \kappa_{n-1} \theta_{n-1}\right) \\
& \times \mathbb{E}_{\lambda_{0}, X}^{t_{n-2}}\left[\exp \left(-\frac{u}{2}\left[\lambda_{n-1} h_{n-1} a_{n-1}+h_{n-2} \lambda_{n-2}+h_{n-1} \lambda_{n-2}\right]\right)\right], \\
= & G_{n-3} \exp \left(-\frac{u}{2} h_{n}^{2} a_{n} \kappa_{n-1} \theta_{n-1}\right) \exp \left(\frac{u^{2}}{8} h_{n-1}^{3} \sigma_{n-2}^{2} \lambda_{n-2} a_{n-1}^{2}\right) \\
& \exp \left(-\frac{u}{2} \lambda_{n-2} h_{n-1} a_{n-1}-\frac{u}{2} \kappa_{n-2}\left(\theta_{n-2}-\lambda_{n-2}\right) h_{n-1}^{2} a_{n-1}-h_{n-2} \lambda_{n-2}-h_{n-1} \lambda_{n-2}\right), \\
= & G_{n-3} \exp \left(-\frac{u}{2} h_{n}^{2} a_{n} \kappa_{n-1} \theta_{n-1}\right) \exp \left(\frac{u^{2}}{8} h_{n-1}^{3} \sigma_{n-2}^{2} \lambda_{n-2} a_{n-1}^{2}\right) \\
& \exp \left(-\frac{u}{2} h_{n-1}^{2} a_{n-1} \kappa_{n-2} \theta_{n-2}\right) \exp \left(-\frac{u}{2} \lambda_{n-2} h_{n-1} a_{n-1}\right. \\
& \left.+\frac{u}{2} \kappa_{n-2} \lambda_{n-2} h_{n-1}^{2} a_{n-1}-h_{n-2} \lambda_{n-2}-h_{n-1} \lambda_{n-2}\right), \\
= & G_{n-3} \exp \left(-\frac{u}{2} h_{n}^{2} a_{n} \kappa_{n-1} \theta_{n-1}-\frac{u}{2} h_{n-1}^{2} a_{n-1} \kappa_{n-2} \theta_{n-2}\right) F_{2}
\end{aligned}
$$




$$
\begin{aligned}
& \times \exp \left(-\frac{u}{2} \lambda_{n-2} h_{n-2}\left[1+\frac{h_{n-1}}{h_{n-2}}+\frac{h_{n-1}}{h_{n-2}} a_{n-1}\left(1-\kappa_{n-2} h_{n-1}\right)\right]\right), \\
= & G_{n-3} \exp \left(-\frac{u}{2} h_{n}^{2} a_{n} \kappa_{n-1} \theta_{n-1}-\frac{u}{2} h_{n-1}^{2} a_{n-1} \kappa_{n-2} \theta_{n-2}\right) \exp \left(-\frac{u}{2} \lambda_{n-2} h_{n-2} a_{n-2}\right) F_{2} .
\end{aligned}
$$

Hence by iterations, we obtain

$$
\begin{aligned}
& \mathbb{E}_{\lambda_{0}, X}^{t_{n-k}}\left[\frac{\exp \left(-u \int_{0}^{T} \lambda_{s} d s\right)}{\prod_{i=1}^{k-1} F_{i}}\right]=G_{n-k-1} \exp \left(-\frac{u}{2} \lambda_{n-k} h_{n-k} a_{n-k}\right) F_{k} \\
& \times \exp \left(-\frac{u}{2} \sum_{i=1}^{k} h_{n-k+i}^{2} a_{n-k+i} \kappa_{n-k+i-1} \theta_{n-k+i-1}\right) .
\end{aligned}
$$

Then until time $t_{0}$, we finally obtain the expected result.

Theorem 3.6. For all $u \in \mathbb{C}$, the conditional Laplace transform $\Phi$ of the regime switching CIR process is given by

$$
\begin{aligned}
& \ln \left(\Phi_{0, T, \lambda, X}(u)\right)=\ln \left(\mathbb{E}_{\lambda_{0}, X}^{t_{0}}\left[\exp \left(-u \int_{0}^{T} \lambda_{s} d s\right)\right]\right) \\
= & -\frac{u}{2} \sum_{k=1}^{n} h_{k}^{2} a_{k} \kappa_{k-1} \theta_{k-1}-\frac{u}{2} h_{1} \lambda_{0}\left[1+a_{1}\left(1-\kappa_{0} h_{1}\right)\right]+\sum_{k=1}^{n} \ln \left(\mathbb{E}_{\lambda_{0}, X}^{t_{0}}\left[C^{2}(k)\right]\right) .
\end{aligned}
$$

where the sequence $a$ is defined in Proposition 3.5 and $C^{2}$ is given by

$C^{2}(k):=\exp \left(\frac{h_{n-k+1}^{3}}{8} u^{2} \sigma_{n-k}^{2} a_{n-k+1}^{2}\left[\lambda_{0}+\sum_{i=0}^{n-k} \kappa_{i}\left(\theta_{i}-\lambda_{i}\right) h_{i+1}+\sum_{i=0}^{n-k} \sigma_{i} \sqrt{\lambda_{i}} \Delta W_{i}\right]\right)$.

Proof. As in [6], we see that it would be difficult to compute the expression $\mathbb{E}_{\lambda_{0}, X}^{t_{n-k-1}}\left[F_{k}\right]$ explicitly. That is why we simply approximate the expression $F_{k}$ at time $t_{n-k}$ by $\mathbb{E}_{\lambda_{0}, X}^{0}\left[F_{k}\right]$. Firstly, we can use the following approximation $\lambda_{n-k} \simeq \lambda_{0}+\sum_{i=0}^{n-k} \kappa_{i}\left(\theta_{i}-\lambda_{i}\right) h_{i+1}+\sum_{i=0}^{n-k} \sigma_{i} \sqrt{\lambda_{i}} \Delta W_{i}$. Then

$$
F_{k}=\exp \left(\frac{h_{n-k+1}^{3}}{8} u^{2} \sigma_{n-k}^{2} a_{n-k+1}^{2}\left[\lambda_{0}+\sum_{i=0}^{n-k} \kappa_{i}\left(\theta_{i}-\lambda_{i}\right) h_{i+1}+\sum_{i=0}^{n-k} \sigma_{i} \sqrt{\lambda_{i}} \Delta W_{i}\right]\right) .
$$

Approximate the expression of $F_{k}$ at time $t_{n-k}$ by the expectation at time 0 , we obtain

$$
\begin{aligned}
\ln \left(\mathbb{E}_{\lambda_{0}, X}^{t_{0}}\left[\frac{\exp \left(-u \int_{0}^{T} \lambda_{s} d s\right)}{\prod_{i=1}^{n-1} F_{i}}\right]\right) & \simeq \ln \left(\mathbb{E}_{\lambda_{0}, X}^{t_{0}}\left[\frac{\exp \left(-u \int_{0}^{T} \lambda_{s} d s\right)}{\prod_{k=1}^{n} \mathbb{E}_{\lambda_{0}, X}^{t_{0}}\left[F_{k}\right]}\right]\right) \\
& =\ln \left(\frac{\mathbb{E}_{\lambda_{0}, X}^{t_{0}}\left[\exp \left(-u \int_{0}^{T} \lambda_{s} d s\right)\right]}{\prod_{k=1}^{n} \mathbb{E}_{\lambda_{0}, X}^{t_{0}}\left[F_{k}\right]}\right) \\
& =\ln \left(\mathbb{E}_{\lambda_{0}, X}^{t_{0}}\left[\exp \left(-u \int_{0}^{T} \lambda_{s} d s\right)\right]\right)-\ln \left(\prod_{k=1}^{n} \mathbb{E}_{\lambda_{0}, X}^{t_{0}}\left[F_{k}\right]\right) \\
& =\ln \left(\Phi_{0, T, \lambda, X}(u)\right)-\sum_{k=1}^{n} \ln \left(\mathbb{E}_{\lambda_{0}, X}^{t_{0}}\left[F_{k}\right]\right) .
\end{aligned}
$$

We conclude using the expression of $F_{k}$. 
Remark 3.1. The analytic approximation formula given in previous Theorem could be also used as a way to simulate conditional Laplace transform.

\section{Simulations}

4.1. Pricing zero coupon Bond in the two firms case with two regimes. We fixe the time maturity $T$ of the zero coupon bond equal to 10 (i.e. a ten years ahead maturity). We take a deterministic risk free interest rate equals to zero.

4.1.1. The model parameters and heuristic. The heuristic of the calculus of the defaultable Bond price is then done by a Monte Carlo approach with $M C \in \mathbb{N}$ steps:

1. We know the value of the infinitesimal generator $\Pi^{X}$ of the credit migration process $\mathrm{X}$. This one is given or estimated using historical data (see Section 4.2).

2. We generate a sequence of increasing stopping times and the corresponding trajectory of the Markov chain X.

3. (a) We apply the formula (6) to calculate the price of this defaultable Bond price for the firm A or B.

(b) We apply the construction of the time grid studied in subsection 3.2.1. Then, we apply the formula (10).

4. We come back to the step 2. until we have done MC times this method.

5. We evaluate the means of the MC values obtained in points 3 (a) and (b).

Hence, assume that we have two regimes which represent a "normal" economic regime (regime 0) and a "crisis" regime (regime 1 ), then the credit migration process $\mathrm{X}$ takes its values in $\{(0,0) ;(1,0) ;(0,1) ;(1,1)\}$. For our simulation, in this part, we fix the transition matrix $P^{X}$ of the credit migration process $\mathrm{X}$ equals to

$$
P^{X}=\left(\begin{array}{cccc}
0.90 & 0.04 & 0.04 & 0.02 \\
0.05 & 0.85 & 0.01 & 0.09 \\
0.05 & 0.01 & 0.85 & 0.09 \\
0.05 & 0.01 & 0.01 & 0.93
\end{array}\right) .
$$

In other words, if we are in a state where only the firm A is on "crisis" (i.e. state $(1,0))$ the probability that the firm B goes into "crisis" in the next time step but the firm A goes back on "normal" economic situation, is 0.01 . Hence, we need to have four different sets of CIR default intensity parameters to model all the states of the economy. Let for $i \in\{A, B\}, \nu^{i}, \xi^{i}$ and $\rho^{i}$ be real valued such that the set of parameters are given by Table 1

\section{Remark 4.1.}

- For $i \in\{A, B\}$, the constant $\nu^{i}, \xi^{i}$ and $\rho^{i}$ are choosen such that the CIR condition holds, i.e. $2 \kappa_{X} \theta_{X} \geq \sigma_{X}^{2}$.

- The state $(0,0)$ can be seen as a standard economic state where nor firm A nor firm B are in crisis. 
TABLE 1. Parameters values of the CIR default intensity in the 2 regimes case.

\begin{tabular}{|c||c|c|c|}
\hline Parameters & $\kappa_{X}$ & $\theta_{X}$ & $\sigma_{X}$ \\
\hline \hline$(0,0)$ & 0.1 & 0.15 & 0.15 \\
\hline$(1,0)$ & $0.1+\nu^{A}$ & $0.15+\xi^{A}$ & $0.15+\rho^{A}$ \\
\hline$(0,1)$ & $0.1+\nu^{B}$ & $0.15+\xi^{B}$ & $0.15+\rho^{B}$ \\
\hline$(1,1)$ & $0.1+\nu^{A}+\nu^{B}$ & $0.15+\xi^{A}+\xi^{B}$ & $0.15+\rho^{A}+\rho^{B}$ \\
\hline
\end{tabular}

4.1.2. Comparison of the different formulas to evaluate defaultable bond price. Convergence: We know that the formula of the conditional survey probability with respect to $\mathbb{G}$ is given by equation (3). We would like now to compare the different formulas to price defaultable zero coupon bond (i.e. formulas (3), (6) and (10)). In tables 2, 3 and 4, we resume the convergence results in the case of a four states regime parameters defined as in Table 1.

TABLE 2. Values of the constant parameters defined in Table 1.

\begin{tabular}{|c||c|c|c|c|c|c|}
\hline Parameters: & $\nu^{A}$ & $\nu^{B}$ & $\xi^{A}$ & $\xi^{B}$ & $\rho^{A}$ & $\rho^{B}$ \\
\hline Values: & 0.2 & 0 & 0 & 0.3 & 0 & 0.1 \\
\hline
\end{tabular}

TABLE 3. Values of the Bond price standard formula in $t=0$ in each regime with a maturity $T=10$ years.

\begin{tabular}{|c||c|c|c|c|}
\hline Regimes: & $(0,0)$ & $(1,0)$ & $(0,1)$ & $(1,1)$ \\
\hline Bond price values: & 0.6086 & 0.3777 & 0.2740 & 0.0668 \\
\hline
\end{tabular}

TABLE 4. Results for the formulas convergence in $t=0$ with initial regime the regime $(0,0)$ and maturity $T=10$ years.

\begin{tabular}{|c||c|c||c|c||c|c|}
\hline Bond Price & Ricatti: 6 (std) & C.T.(sec.) & Analytic: 10 (Std) & C.T. & MC: 3 & C.T. \\
\hline$M C=100$ & $0.5619(0.1110)$ & 1.94 & $0.5585(0.1699)$ & 15.98 & 0.6500 & 1.95 \\
\hline$M C=300$ & $0.5692(0.1015)$ & 5.34 & $0.5587(0.1602)$ & 52.52 & 0.6233 & 6.61 \\
\hline$M C=400$ & $0.5736(0.0949)$ & 6.87 & $0.5649(0.1505)$ & 60.48 & 0.6400 & 9.58 \\
\hline$M C=500$ & $0.5748(0.0927)$ & 7.97 & $0.5658(0.1511)$ & 78.32 & 0.6360 & 12.44 \\
\hline$M C=1000$ & $0.5738(0.0961)$ & 16.31 & $0.5654(0.1533)$ & 146.51 & 0.6220 & 33.23 \\
\hline$M C=2000$ & $0.5727(0.0995)$ & 27.33 & $0.5646(0.1533)$ & 221.22 & 0.5770 & 96.78 \\
\hline
\end{tabular}

Remark 4.2. We take for the time step parameter $\Delta_{t}$ (appearing in subsection 3.2 .1 for the calculus of (10)) the value 0.01. Since we obtain the following Bond prices: 0.5612 with $\Delta_{t}=1,0.5648$ with $\Delta_{t}=0.1$ and 0.5649 with $\Delta_{t}=0.01$

In Table 4, we can see that all formulas converge when the number of Monte Carlo simulations increases. Whereas the bond price value given by formula (6) based on Riccati approach or formula (10) based on analytic approach converges quicker than the value given by formula (3). Indeed, it is sufficient to take 400 Monte Carlo simulations, for formulas (6) and (10), to 
converge while it is necessary to take at least 2000 Monte Carlo simulations with formula (3). The difference of $10^{-1}$ on the value given by (6) and (10) could be due to the approximation error of the conditional expectation at time $t_{n-k}$ of $F_{k}$ (see. proof of Theorem 3.6). Hence, our two formulas need less simulations than formula (3) to converge. Moreover we observe that the Riccati approach formula (6) need a smaller computation time. Only $6.87 \mathrm{sec}$ while formula (10) needs $60.48 \mathrm{sec}$ and formula (3) needs $96.78 \mathrm{sec}$. Hence formula based on Riccati approach needs ten times less times than Analytic approach to converge. Whereas, we know that we used CIR model for intensity modeling since there exists explicit formula for bond. Hence as we said before, the analytic approach could be interesting if we would like to obtain an explicit easy scheme to simulate defaultable bond.

Bond price with respect to the maturity $\mathrm{T}$ :

We observe in Table 5 that the three formulas give similar results. Whereas, firstly, we made these simulations taking 2000 Monte Carlo simulations for the Probabilistic approach (formula (3)). Secondly, we remark, when the maturity $\mathrm{T}$ is greater than 10 , that the result given by the analytic approximation is not better than the other. This relative mispricing was observed in the non regime switching case and uniform step time model discretization in [6] as soon as the maturity $\mathrm{T}$ is greater than 10 .

TABLE 5. Value of the Defaultable zero coupon bond price at time 0 with respect to the maturity time $T$ with $\Delta_{t}=0.01$.

\begin{tabular}{|c||c|c|c|}
\hline Bond Price & Ricatti: $6(M C=400)$ & Analytic: $10(M C=400)$ & MC: $3(M C=2000)$ \\
\hline$T=1$ & 0,9926 & 0,9923 & 0,9940 \\
\hline$T=2$ & 0,9709 & 0,9696 & 0,9770 \\
\hline$T=5$ & 0,8458 & 0,8405 & 0,8480 \\
\hline$T=7$ & 0,7376 & 0,7261 & 0,7365 \\
\hline$T=10$ & 0,5736 & 0,5649 & 0,5770 \\
\hline$T=15$ & 0,3579 & 0,3948 & 0,3505 \\
\hline
\end{tabular}

4.1.3. Bond Price in function of the probability that $B$ goes to crisis. Taking parameters as in Table 2, we evaluate the price of a defaultable zero coupon bond in function of the probabilities $P\left(X_{t+\Delta_{t}}=(0,1) \mid X_{t}=(0,0)\right)$ and $P\left(X_{t+\Delta_{t}}=(1,1) \mid X_{t}=(0,0)\right)$. These are $p_{1,3}^{X}$ and $p_{1,4}^{X}$. Hence, we take a parametric transition matrix of the form:

$$
P^{X}=\left(\begin{array}{cccc}
1-a-3 b & a & 2 b & b \\
0.05 & 0.85 & 0.01 & 0.09 \\
0.05 & 0.01 & 0.85 & 0.09 \\
0.05 & 0.01 & 0.01 & 0.93
\end{array}\right)
$$

where $a, b \in[0,1]$.

The result is stated in Figure 1. We observe when b grows up, which is the probability $P\left(X_{t+\Delta_{t}}=(1,1) \mid X_{t}=(0,0)\right)$, that the price of the defaultable zero coupon bond price of the firm A decreases. This means that the economic status of the firm B (the probability to go in crisis) impacts the value of the defaultable zero coupon bond of the firm A.

4.2. Regime switching defaultable intensity estimation. We work now on real data. We are interesting now in the modeling issue. We will show that our regime switching model captures well some market features or economics behavior. Hence, we can use this algorithm to estimate the intensities of the two firms and then construct the Markov copula as explained in Section 2. 


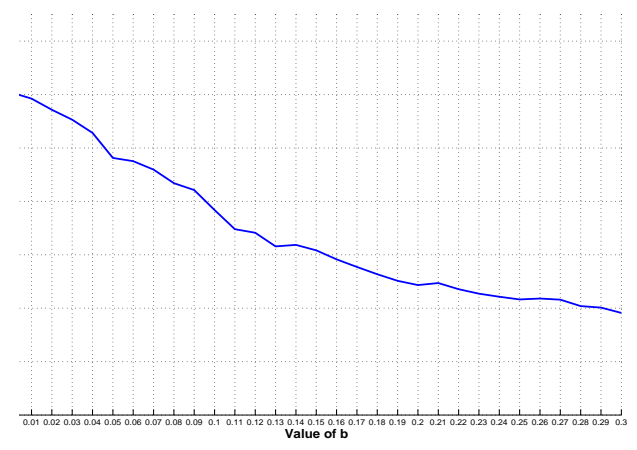

Figure 1. Price of a defaultable zero coupon bond price in $t=0$ for maturity $T=10$ and values of $a=0.04$ in function of $b$.
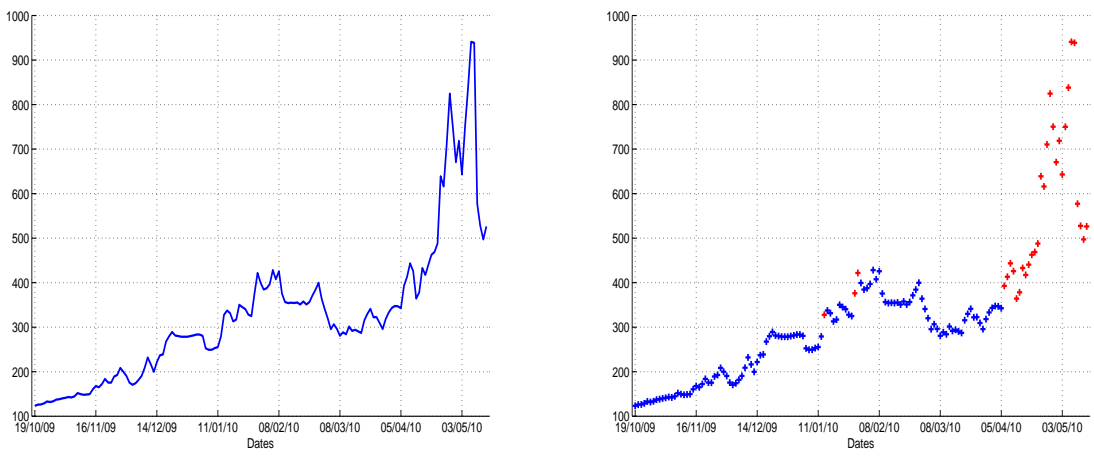

FiguRE 2. Greek Spread between the $19 / 10 / 2009$ to $13 / 05 / 2010$. On right, the estimation result (The color blue is when we are in regime 1 and red for regime 2).

4.2.1. Estimation on Greek sovereign spread between 19/10/2009 to $13 / 05 / 2010$. Firstly, the Figure 2, left, shows the plot of the Greek sovereign spread between 19/10/2009 and 13/05/2010. For the estimation, we use the estimation procedure developed and studied in Goutte and Zou [15] for regime switching Cox Ingersoll Ross process applied to foreign exchange rate data. Moreover, we assume that there are two regimes. This means that there is a "good" one and a "bad" one economies like a time crisis period and a "standard" economic period, or a spike time period and a non spike time period. Our results are stated in Table 4.2.1. Figure 2, right, gives a graphical representation of the states classification obtained by this procedure.

4.2.2. Interpretations. We can see clearly in Figure 2, right, that there are two significantly different time periods. The first one between the 19/10/09 and april 2010, and the second one after april 2010. The second one corresponds to the beginning of the economic world crisis. Hence, we can see on the estimation results in Table 4.2.1 that parameters values are very 
TABLE 6. Maximum Likelihood estimation results.

\begin{tabular}{|c||c|c|c|c|c|}
\hline & $\hat{\kappa}$ & $\hat{\theta}$ & $\hat{\sigma}$ & $\hat{\Pi}_{i i}^{X}$ & $\pi_{i}$ \\
\hline \hline Regime 1 & 0.022860 & 309.460660 & 0.774675 & 0.974977 & 0.723722 \\
\hline Regime 2 & 0.117918 & 620.721205 & 3.092136 & 0.934452 & 0.276278 \\
\hline
\end{tabular}
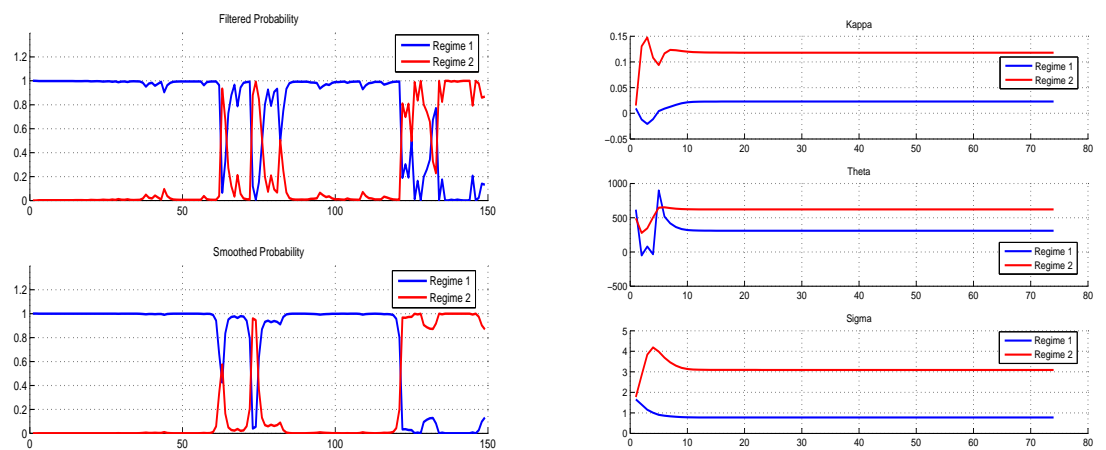

FiguRE 3. On left: Smoothed and Filtered probabilities. On right: Parameters convergence steps

different in each regime. Before the crisis, we have a mean reverting parameter less than after crisis, $\hat{\kappa_{1}}=0.02286$ against $\hat{\kappa_{2}}=0.117918$. And in the time crisis period the volatility of the defaultable intensity is multiplied by 4 with respect to the volatility value before crisis. It is an expected result since we are in a crisis period so the risk of a firm to make default is higher than in a standard economic situation.

We can also see in the right graph of Figure 3, that the estimation process is fast. Indeed only 15 iterations of our algorithm are sufficient for convergence to the true estimated parameters values.

Moreover, to evaluate the good fit classification of our model, we can calculate the Regime classification measure (RCM) obtained by this regime switching model. In fact, let $K>0$ be the number of regimes, the RCM statistics is then given by

$$
R C M(K)=100\left(1-\frac{K}{K-1} \frac{1}{T} \sum_{t=1}^{T} \sum_{i=1}^{K}\left(P\left(X_{t}=i \mid \mathcal{F}_{T}^{\lambda} ; \hat{\Theta}\right)-\frac{1}{K}\right)^{2}\right),
$$

where the quantity $P\left(X_{t}=i \mid \mathcal{F}_{T}^{\lambda} ; \hat{\Theta}\right)$ is the smoothed probability given in the left graph on Figure 3 and $\hat{\Theta}$ is the vector parameter estimation results (i.e. $\left.\hat{\Theta}:=\left(\hat{\kappa}, \hat{\theta}, \hat{\sigma}, \hat{\Pi}^{X}\right)\right)$. The constant serves to normalize the statistic to be between 0 and 100. Good regime classification is associated with low RCM statistic value: a value of 0 means perfect regime classification and a value of 100 implies that no information about regimes is revealed. In our case we obtain a RCM equals to 8.41. Hence, it shows that this model with regime switching parameters captures very well two significant economics time period. And so this is a real add for the valuation of defaultable bond.

4.2.3. Methodology. Hence, we can apply this estimation method to find each estimated parameters for firms or countries A and B. Then, using the copula construction theory defined in Corollary 2.2 and developed in section 2.2.1, we can apply the pricing formulas given in 
Theorem 2.7 to obtain the price of defaultable bond with respect to the correlation regime structure of each defaultable intensity regime switching estimations.

\section{Acknowledgements}

The authors are grateful to Huyên Pham for his stimulating remarks and comments which allowed us to improve this paper.

\section{References}

1. A. Alfonsi and D. Brigo, Credit default swap calibration and derivatives pricing with the SSRD stochastic intensity model, Finance Stochastic 9 (2005), 29-42.

2. T. R. Bielecki, J. Jakubowski, A. Vidozzi and L. Vidozzi, Study of dependence for some stochastic processes, Stoch. Anal. Appl. 26 no. 4 (2008), 903-924.

3. T. R. Bielecki, M. Jeanblanc and M. Rutkowski, Credit risk, Lecture Note of Lisbonn. http://www.maths.univ-evry.fr/pages_perso/jeanblanc/conferences/lisbon.pdf. (2006)

4. T.R. Bielecki and M. Rutkowski, Credit Risk: Modeling, Valuation and Hedging, Springer 2002.

5. S. Choi, Regime-Switching Univariate Diffusion Models of the Short-Term Interest Rate, Studies in Nonlinear Dynamics \& Econometrics, 13, No. 1, Article 4 (2009).

6. Y. Choi and T.S. Wirjanto, An analytic approximation formula for pricing zero-coupon bonds, Finance Research Letters, 4, (2007), 116-126.

7. J.C. Cox, J. Ingersoll and S. Ross, A theory of the term structure of interest rates, Econometrica, 53 (1985), 385-405.

8. D. Duffie, D. Filipovic and W. Schachermayer, Affine processes and applications in finance, Ann. Appl. Probab. 13, Number 3, (2003) 984-1053.

9. D. Duffie and K. Singleton, Credit risk: Pricing, Measurement and Management, Princeton University Press, Princeton 2003.

10. E. Eberlein and F. Ozkan, The defaultable Lévy term structure: ratings and restructuring, Mathematical Finance 13 (2003), 277-300.

11. R.J. Elliott, L. Aggoun and J.B. Moore, Hidden Markov Models: Estimation and Control, Springer-Verlag, New York 1995.

12. N. El Karoui, M. Jeanblanc and Y. Jiao, What happens after a default: the conditionnal density approach, Preprint (2009).

13. Z. Grbac, Credit Risk in Levy Libor Modeling: Rating Based Approach, Dissertation. University Freibourg. http://www.freidok.uni-freiburg.de/volltexte/7253/pdf/diss.pdf (2009).

14. C. Gourieroux, Continuous time Wishart process for stochastic risk, Econometric Reviews, 25 (2-3) (2006), 177-217.

15. S. Goutte and B. Zou, Continuous time regime switching model applied to foreign exchange rate, Preprint (2012).

16. J. Hamilton, Rational-expectations econometric analysis of changes in regime, Journal of Economic Dynamics and Control, 12 (1989), 385-423.

17. J.M. Harrison and S.R. Pliska, Martingales and stochastic integrals in the theory of continuous trading, Stoch. Proces. Appl. 11 (1981), 381-408.

18. J.M. Harrison and S.R. Pliska, A stochastic calculus model of continuous time trading: complete markets, Stoch. Proces. Appl. 13 (1983), 313-316.

19. S. Heston, A closed-Form Solution for Options with Stochastic Volatility with Application to Bond and Currency Options, Review of Financial Studies, 6 (1993), 327-343.

20. M. Jeanblanc and M. Rutkowski, Mathematical Finance-Bachelier Congress 2000, Chapter Default risk and hazard process, 281-312, Springer-Verlag Berlin 2002.

21. D. Lando, On Cox Processes and Credit Risky Securities, Review of Derivatives Research, 2 (1998), 99-120. 
22. D. Madan and H. Unal, Pricing the risks of default, Review of Derivatives Research 2 (1998), 121-160.

23. R. Merton, On the pricing of corporate debt: the risk structure of interest rates, J. of Finance, 3 (1974), 449-470.

Stéphane Goutte received M.Sc. Paris at XIII University and Ph.D at University of Paris XIII (France) and LUISS Roma (Italy). Since 2010, he is a researcher at Centre National de la Recherche Scientifique (CNRS) in University Paris 7 Diderot. His research interests include mathematical finance, stochastic calculus, stochastic modelling applied on economy and computational mathematics.

Laboratoire de Probabilités et Modèles Aléatoires (LPMA), Université Paris Diderot, 175 rue de Chevaleret, 75013 Paris France.

e-mail: goutte@math.univ-paris-diderot.fr

Armand Ngoupeyou received M.Sc. from Paris Dauphine University, and Ph.D. from Evry University (France). Since 2010, he is a researcher at ALMA Research. His research interests are mathematical finance and credit risk.

Laboratoire de Probabilités et Modèles Aléatoires (LPMA), Université Paris Diderot, 175 rue de Chevaleret, 75013 Paris France.

e-mail: armand.ngoupeyou@univ-paris-diderot.fr 